\title{
Bone Marrow Dyspoiesis and Revised International Prognostic Scoring System- A Comparative Study
}

\author{
Kiran Sapruํ, Geeta Vidyadharan², Neeraj Sidharthan ${ }^{3}$ \\ ${ }^{1}$ Assistant Professor, Department of Pathology, Amrita Institute of Medical Sciences, Kochi, Kerala, India. \\ ${ }^{2}$ Associate Professor, Department of Pathology, Amrita Institute of Medical Sciences, Kochi, Kerala, India. \\ ${ }^{3}$ Associate Professor, Department of Pathology, Amrita Institute of Medical Sciences, Kochi, Kerala, India.
}

\section{ABSTRACT}

\section{BACKGROUND}

Myelodysplastic syndrome (MDS) can be defined as a clonal haematopoietic stem cell disorder characterized by ineffective haematopoiesis and leukemia progression. It represents the most common cause for bone marrow failure in adults. We wanted to compare the extent of dyspoiesis and RIPSS in assessing the risk score in MDS patients.

\section{METHODS}

This was a comparative study conducted in Amrita Institute of Medical Science, which included 4 years of retrospective and 2 years and 8 months of prospective data of patients who were Kipp diagnosed as MDS. Scoring was attempted by assessing morphological features of RBCs, WBCs and megakaryocytes. The morphology of these cases was reviewed, grouped and the findings in the two groups were compared with respect to the R- IPSS categories on 54 patients.

\section{RESULTS}

Morphological scoring was done on the extent of dyspoiesis. Number of cases in which dyspoiesis was below the mean value was 33 , and were assigned as low risk group which comprised the majority, amounting to 18/33. In high risk and very high-risk groups we had 4 patients each. The cases with scores above the mean value were 3 in number among high risk and 3 in very high risk. We also had 7 cases each in low and intermediate grades and 1 case among the very low grade. From the data assessed, no concordance could be found between the morphological scoring and R- IPSS.

\section{CONCLUSIONS}

There was a significant number of patients in high risk and very high-risk group having a morphological score which was less than the mean value. This emphasizes the significance of assessing the morphology along with cytogenetic findings giving equal importance to both in all cases of MDS.

\section{KEY WORDS}

Dysplasia, Unilineage, Multilineage, Refractory, Cytogenetics
Corresponding Author: Dr. Geeta Vidyadharan, Associate Professor, Department of Pathology, Amrita Institute of Medical Sciences, Kochi, Kerala, India.

E-mail: keerthanageeta@gmail.com

DOI: $10.14260 / \mathrm{jemds} / 2019 / 682$

Financial or Other Competing Interests: None.

How to Cite This Article:

Sapru K, Vidyadharan G, Sidharthan N. Bone marrow dyspoiesis and revised international prognostic scoring system- a comparative study. J. Evolution Med. Dent. Sci. 2019;8(42):3146-3150, DOI: $10.14260 / \mathrm{jemds} / 2019 / 682$

Submission 24-05-2019, Peer Review 04-10-2019, Acceptance 11-10-2019, Published 21-10-2019. 


\section{BACKGROUND}

Myelodysplastic syndrome is a clonal haematopoietic stem cell disorder characterized by ineffective haematopoiesis and leukemic progression. ${ }^{[1]}$ It represents the most common cause of bone marrow failure in adults. Incidence of MDS occur in 5 per 100,000 people. In older individuals more than 70 years, the incidence increases to between 22 and 45 per 100,000 and increases further with age. Collectively, the term MDS describes a diverse group of clonal disorders of haematopoietic stem or progenitor cells. MDS comprises a group of clonal disorders of haematopoietic stem or progenitor cells resulting in ineffective haematopoiesis, abnormal "dysplastic" cell morphology, and potential for clonal evolution. ${ }^{[2]}$ MDS have been referred to as oligoblastic leukemia, refractory anaemia, smouldering acute leukemia, or preleukemia. They are considered as preleukemic conditions with a greater risk of progression to acute myeloid leukemia (AML).[3] The natural history of these diseases ranges from a chronic course that may span years to a rapid course toward leukemic progression. WHO tumours of haematopoietic and lymphoid tissues, 2008 defines evolution to secondary acute myeloid leukemia in MDS patients as $20 \%$ myeloid blasts in the blood or marrow, or the presence of one of several AML defining karyotypic abnormalities [e.g.; $t$ (15;17), $t$ (8;21), inv $(16)$, ort $(16 ; 16)]$ regardless of blast proportion. MDS can develop both as de novo and as a consequence of previous chemo- or radiotherapy in cancer patients. MDS is considered a disease related to aging. [2] Myelodysplastic syndromes is very uncommon in children accounting for less than $5 \%$ of all haematopoietic neoplasms in patients less than 14 years of age. The denovo or primary form of MDS in children should be distinguished from cases of secondary MDS that follow congenital or acquired BM failure syndromes and therapy related MDS that follow cytotoxic therapy for a previous neoplastic or non- neoplastic condition. Furthermore, although MDS associated with Down's syndrome has been reported to account for $20-25 \%$ of cases of childhood MDS in the past, this disorder is now considered as a unique biologic entity synonymous with Down syndrome related myeloid leukemia and distinct from other cases of childhood MDS.

In paediatric population of MDS patients, inherited bone marrow-failure syndromes like Fanconi anaemia, Shwachman-Diamond syndrome, severe congenital neutropenia, dyskeratosis congenital, and Diamond-Black fan anaemia, are related high-risk factors. In myelodysplastic syndromes (MDS) different prognostic risk analysis systems based on clinical and morphological data are used for predicting survival..[4]

Many morphological, immunophenotypic and genetic features observed in MDS in adults are also seen in childhood forms of the disease, but there are some significant differences. The specific identification and quantification of dysplasia is critical for a correct application of the WHO classification because in MDS with fewer than $5 \%$ bone marrow blasts, the WHO proposal introduced uni-versus multilineage dysplasia as a diagnostic criterion which in turn helps in increasing the prognostic value of the classification; the finding, of dysplastic morphological alterations in at least $10 \%$ of the cells of at least one myeloid lineage is the most important criterion for the diagnosis of RCUD, in an appropriate clinical setting. Because of the minimal percentage of blasts in the bone marrow and the low incidence of chromosome abnormalities this subtype is rather difficult to identify. In various haematological, as well as nonhaematological disorders, variable degrees of dyserythropoiesis are commonly observed of which the morphological abnormalities of the granulocytic and megakaryocytic series are more specific and significant for the diagnosis of MDS. However, no single morphological finding is diagnostic for MDS that sometimes remains a diagnosis of exclusion.

For children the same criteria used for adults with refractory anaemia with excess blasts should be applied. Currently, in contrast to adult MDS, there are no available studies that have investigated the prognostic significance of distinguishing RAEB-1 and RAEB-2 in children. Some cases diagnosed in children as acute myeloid leukemia with 20$29 \%$ blasts in the PB and or BM that have myelodysplasia related changes, including cases with MDS related cytogenetic abnormalities may also have slowly progressive disease. These cases considered as refractory anaemia with excess blasts in transformation in the FAB classification, may lack the clinical features of acute leukemia and behave more like MDS than AML. Children who present with a peripheral blood and or BM disorder associated with $t(8 ; 21)$ (q22;q22), inv (16) (p13.1q22) or t(16;16) (p13.1;q22) or $t$ $(15 ; 17)$ (q22;q12) should be considered to have AML regardless of the blast count.[5]

Initial evaluation of patients with suspected MDS requires careful assessment of their peripheral blood smear and blood counts, marrow morphology, duration of their abnormal blood counts, other potential causes for their cytopenias, and concomitant illnesses. The FrenchAmerican-British (FAB) classification initially categorized patients for the diagnostic evaluation of MDS. Dysplastic changes in at least 2 of the 3 haematopoietic cell lines have been used by most histopathologists to diagnose MDS. These changes include megaloblastoid erythropoiesis, nucleocytoplasmic asynchrony in the early myeloid and erythroid precursors, and dysmorphic megakaryocytes. Patients with MDS are classified as having 1 of 5 subtypes of disease: refractory anaemia (RA); RA with ringed sideroblasts (RARS); RA with excess of blasts (RAEB); RAEB in transformation (RAEB-T); or chronic myelomonocytic leukemia (CMML). MDS are generally indolent, with patients' blood counts remaining relatively stable over at least several months.[6] The diagnosis of MDS is typically made by excluding other non MDS causes of cytopenias in the presence of some combination of dysplastic cell morphology, increased marrow blasts and a karyotypic abnormality. Common "MDS mimics" include cytopenias or morphologic abnormalities as a result of a medication (eg, Methotrexate); deficiencies of cobalamin, folate, or copper; excessive alcohol use; HIV infection; immune-mediated cytopenias, including aplastic and large granular lymphocyte leukemia; congenital syndromes such as Fanconi and X-linked sideroblastic anaemia; and other neoplasms such as myeloproliferative neoplasms. Bone marrow aspirate and biopsy allows for assessment of both cell morphology and histological architecture and when coupled with conventional karyotyping (Abnormal in approximately one-half of de novo MDS cases and in $80 \%$ of cases arising secondary to exposure to a DNA damaging agent), can in some cases 
confirm disease clonality. Morphologic dysplasia is not required for an MDS diagnosis in the presence of cytopenias if either excess blasts in the $5 \%$ to $19 \%$ range or evidence of clonality restricted haematopoiesis are present.

The International Prognostic Scoring System (IPSS) was created for evaluating clinical outcomes of patients with myelodysplastic syndromes (MDS).The International Prognostic Scoring System (IPSS) is an important standard tool for assessing prognosis of primary untreated adult patients with myelodysplastic syndromes (MDS). The International Prognostic Scoring System consists of blast percentage, karyotype, and number of cytopenias to generate a scoring system which will help to estimate the survival and risk of transformation to acute myeloid leukemia for patients with MDS. The threshold for cytopenias as recommended in the International Prognostic Scoring System (IPSS) for risk stratification in the MDS are Haemoglobin $<10 \mathrm{~g} / \mathrm{dl}$, absolute neutrophil count (ANC) $<1.8 \times 109 / \mathrm{L}$ and platelets $<100 \times 109 /$ L. Values above these thresholds are however, not exclusionary for a diagnosis of MDS, if definitive morphologic and/or cytogenetic findings are present $(1,7)$. The dysplasia may be accompanied by an increase in myeloblasts in the peripheral blood and bone marrow but the number usually $<20 \%$ which is the requisite threshold recommended for the diagnosis of AML (1). This scoring system is universally accepted and is often combined with FAB or World Health Organization morphologic criteria to provide a more complete clinical picture and the most accurate prognostic assessment possible. A detailed cytogenetic analysis is neither feasible and nor easily available even in a tertiary care hospital. In the present study RIPSS was followed which included cytogenetics, bone marrow blast percentage, haemoglobin, platelet and absolute neutrophil count. The main modification in RIPSS compared to IPSS is inclusion of haemoglobin, platelet count and absolute neutrophil count in place of cytopenias ${ }^{[8,9]}$ and in our study we have analysed the extend of dyspoiesis and assessed whether it is comparable with RIPSS scoring system. Determination of the erythrocyte sedimentation rate and lactate dehydrogenase level can establish the presence of an acute process or rapid cell turnover.[10]

\section{METHODS}

This was a comparative study conducted in a Department of pathology, Amrita Institute of Medical Sciences, Kochi for a period of 2 years and 8 months in 54 patients who was diagnosed as MDS, having clinical picture, morphological dysplasia with cytogenetic data. Based on the results obtained on the percentage of subjects diagnosed with MDS multilineage dysplasia from an earlier publication and with $99 \%$ confidence and $15 \%$ allowable error the minimum sample comes to 100 . However, as we could include only those patients who had a detailed cytogenetic data available, our numbers was reduced to 54 .

Ethical clearance was obtained, and clearance number is Dissertation review/MD/MS/2013/5.

Sample collection was done from pathology archives which included 4 years of retrospective and 2 years and 8 months of prospective data. All bone marrow aspirate was stained by Giemsa and Leishman stains. Revised International Prognostic Scoring System scoring system was used initially to assess the risk score. Morphological Scoring was done. Erythroid series cells were evaluated for megaloblastoid change, bi/multinuclearity, nuclear lobation or irregular contours, pyknosis, cytoplasmic fraying and ringed sideroblast. Myeloid cells were assessed on the basis of myeloblasts, Auer rods, pseudo Pelger-Huet abnormality, abnormal nuclear shapes and neutrophil hypogranulation. Dysmegakaryopoiesis was evaluated using micromegakaryocytes, small binucleated megakaryocytes, megakaryocytes with multiple separated nuclei and hypolobated/ monolobated megakaryocytes and weighted scores were given.[2] We included all patients diagnosed as MDS and who had their clinical evaluation, bone marrow aspiration and cytogenetic evaluation done. Patients who didn't have these parameters fulfilled were excluded from this study. Morphological scoring of these cases were reviewed. The comparison of scoring of morphological dyspoiesis and IPSS scoring was done on 54 patients and assessed in detail. Individual total score was assessed for each patient and the mean average of the scores were taken.

\section{Statistical Analysis}

By Chi square test for the association of degree of MDS with demographic and clinical features, was done.

\section{RESULTS}

An association was attempted on the basis of morphological scoring, [2] and RIPSS scoring done on the 54 patients. The parameters analysed and their morphologic changes in RBC (Table/Fig. 1), WBC (Table/ Fig. 2) and megakaryocytic 3 series (Table/ Fig. 3) were assessed.

\begin{tabular}{|c|c|}
\hline Dyspoietic Features & Number of Cases \\
\hline Nuclear budding & 10 \\
\hline Intranuclear bridging & 2 \\
\hline Karyorrhexis & 6 \\
\hline Multinuclearity & 19 \\
\hline Hyperlobation & 1 \\
\hline Megaloblastoid changes & 47 \\
\hline Ring sideroblast & 1 \\
\hline Vacuolation & 1 \\
\hline Nucleocytoplasmic asynchrony * & 32 \\
\hline Nuclear irregularity* & 63 \\
\hline Cytoplasmic blebbing & 6 \\
\hline Table 1. Dyspoietic Features in $\mathbf{R B C}$ \\
\hline *not included in who 2008
\end{tabular}

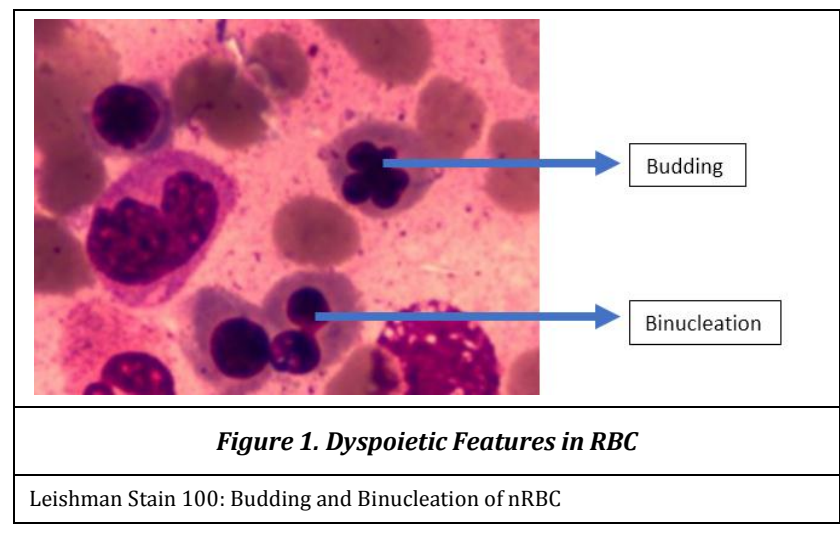




\begin{tabular}{|c|c|}
\hline Dyspoietic Features & Number of Cases \\
\hline Hypolobation & 37 \\
\hline Hypersegmentation & 1 \\
\hline Decreased granules & 15 \\
\hline Auer rods & 1 \\
\hline Ringed neutrophil* & 3 \\
\hline Hypergranulation * & 5 \\
\hline \multicolumn{2}{|c|}{ Table 2. Dyspoietic Features in WBC } \\
\hline *Not included in WHO 2008
\end{tabular}

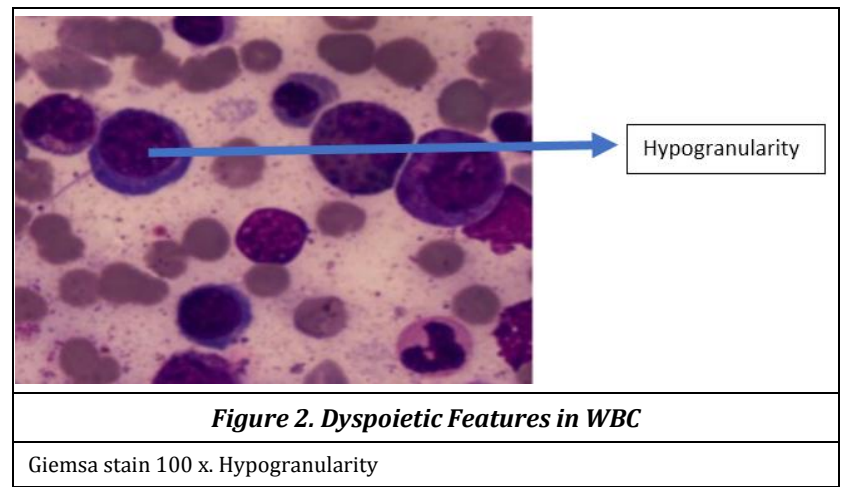

\begin{tabular}{|c|c|}
\hline Dyspoietic Features & Number of Cases \\
\hline Micromegakaryocytes & 10 \\
\hline Nuclear hypolobation & 21 \\
\hline Multinucleation & 7 \\
\hline Hypogranulation* & 1 \\
\hline \multicolumn{2}{|c|}{ Table 3. Dyspoietic Features in Megakaryocytes } \\
\hline * Not included in WHO 2008 \\
\hline
\end{tabular}

\section{DISCUSSION}

Myelodysplastic syndromes (MDS) are a group of clonal disorders arising from haematopoietic stem cells generally characterized by inefficient haematopoiesis, dysplasia in one or more myeloid cell lineages, and variable degrees of cytopenias.[11,12] The MDS differ from many other haematologic malignancies in their chronicity and the morbidity and mortality caused by chronic cytopenias. MDS usually shows cytopenia, mainly due to early death of partially or fully differentiated haematopoietic stem cells and insufficient differentiation capacity of the progenitors to mature blood cells. Deficiencies in haematopoiesis lead to cytopenias, which may vary in severity. Not surprisingly, morbidity and mortality result from bleeding, and infection, along with transformation to AML, which occurs in approximately one-third of patients.(1)

If MDS is suspected, a bone marrow biopsy and aspirate should be performed. The diagnosis is made based on cell morphology, immunohistochemistry, and, in the case of more advanced subtypes of MDS, flow cytometry. Obtaining a bone marrow aspirate sample for cytogenetics is crucial, because abnormalities may help in establishing the diagnosis and in risk stratification. ${ }^{[13]}$

The prognosis of MDS, which differs among FAB subtypes and is more accurately predicted by the International Prognostic Scoring System (IPSS), is extremely poor. WHO classification (2008) of primary myelodysplastic syndromes provides a very good diagnostic tool, improves risk stratification and helps to identify patients for different treatment strategies. The aim of the classification is to define MDS types with different prognoses.[14]

A distinction is made between refractory cytopenia with unilineage dysplasia (RCUD) and refractory anaemia with ring sideroblasts (RARS) as entities in which the signs of dysplasia are limited to erythropoiesis, and refractory cytopenia with multilineage dysplasias (RCMD), in which two or, usually, three cell lineages are dysplastic. Porta $\mathrm{M}$ et al ${ }^{[13]}$ developed scoring system to define minimal morphological criteria associated to the presence of marrow dysplasia. In this study the authors showed the scoring had high sensitivity/specificity ( $>90 \%$ ). The severity of granulocytic and megakaryocytic dysplasia significantly affected survival. According to Valeria $\mathrm{V}$ almost $78 \%$ of patients carry at least 1 mutation which forms the basis for response to therapies.[15]

There was a significant number of patients in high risk and very high-risk groups having a morphological score less than that of the mean value, moreover significant number of low risk grade patients had high mean value. While analysing the cytogenetic data of these subjects it was found that few having low morphological score had significant cytogenetic abnormality which put them in a high-risk R-IPSS scoring system and vice versa. This emphasizes the importance of cytogenetic in assessing risk stratification in MDS patient.[16,17,18]

\section{Limitations}

The main limitations we faced in our study was that cytogenetic analysis could not be done in detail in all patients due to their financial constraints, the selection of cases was mainly from haematology archives, those patients with subtle dyspoiesis at the time of presentation and later diagnosed as MDS by cytogenetic methods would have been missed. As our study was a novel study, comparison with other similar studies was not possible even after extensive search limiting our statistical analysis.

\section{CONCLUSIONS}

From the data assessed by us, no concordance could be found between the morphological scoring and R-IPSS. There was a significant number of patients in high risk and very high-risk group having a morphological score of less than that of the mean value; moreover, significant number of low risk grade patients had high mean value. Morphology alone is often insufficient to reach a final diagnosis, it should be integrated, with other advanced investigations such as flow cytometry, molecular studies, in vitro culture of haematopoietic progenitors.

\section{REFERENCES}

[1] Li J. Myelodysplastic syndrome haematopoeitic stem cell. Int J Cancer 2013;133(3):525-33.

[2] Invernizzi R, Quaglia F, Porta MGD. Importance of classical morphology in the diagnosis of myelodysplastic syndrome. Mediterr J Hematol Infect Dis 2015;7(1):e2015035.

[3] Albitar M, Manshouri T, Shen Y, et al. Myelodysplastic syndrome is not merely "Preleukemia". Blood 2002;100(3):791-8. 
[4] Maes B, Meeus P, Michaux L, et al. Application of the International Prognostic Scoring System for myelodysplastic syndromes. Ann Oncol 1999;10(7):8259.

[5] Estey E, Thall P, Beran M, et al. Effect of diagnosis (refractory anaemia with excess blasts, refractory anaemia with excess blasts in transformation, or acute myeloid leukemia [AML]) on outcome of AML-type chemo-therapy. Blood 1997;90(8):2969-77.

[6] Harris NL, Jaffe ES, Diebold J, et al. The World Health Organization classification of haematological malignancies: report of the Clinical Advisory Committee Meeting, Airlie House, Virginia, November 1997. Mod Pathol 2000;13(2):193-207.

[7] Germing U, Strupp C, Kuendgen A, et al. Prospective validation of the WHO proposals for the classification of myelodysplastic syndromes. Hematologica 2006;91(12):1596-604.

[8] Bain BJ, Clark DM, Wilkins BS, et al. Metastatic tumors. In: Bain BJ, Clark DM, Wilkins BS, eds. Bone Marrow Pathology. $3^{\text {rd }}$ edn. England, Malden, MA: Blackwell Science, Bon Oxford Publication 2001: p. 430-61.

[9] Greenberg LP, Tuechler H, Schanz J, et al. Revised international prognostic scoring system for MDS. Blood 2012;120(12):2454-65.

[10] Passamonti F, Mora B, Maffioli M. New molecular genetics in the diagnosis and treatment of myeloproliferative neoplasms. Curr Opin Hematol 2016;23(2):137-43.

[11] Swerdlow SH, Campo E, Pileri SA, et al. The 2016 revision of world health organization classification of lymphoid neoplasms. Blood 2016;127(20):2375-90.
[12] Porta DMG, Travaglino E, Boveri E, et al. Minimal morphological criteria for defining bone marrow dysplasia: a basis for clinical implementation of WHO classification of myelodysplastic syndrome. Leukemia 2015;29(1):66-75.

[13] Codispoti KET, Depalma L. Myelodysplastic syndrome in elderly patients: correlation of $\mathrm{CBC}$ with cytogenetic and FISH analysis. Int J Lab Hematol 2010;32(4):443-8.

[14] Lee JH, Lee JH, Shin YR, et al. Application of different prognostic scoring systems and comparison of the FAB and WHO classifications in Korean patients with myelodysplastic syndrome. Leukemia 2003;17(2):30513.

[15] Valeria V, Tiu RV, Rogers HJ. Pathogenesis of myelodysplastic syndromes: an overview of molecular and non-molecular aspects of the disease. Blood Research 2014;49(4):216-27.

[16] Malcovati L, Hellström-Lindberg E, Bowen D, et al. Diagnosis and treatment of primary myelodysplastic syndromes in adults. Recommendations from the European LeukemiaNet. Blood 2013;122(17):2943-64.

[17] Malcovati L, Porta DMG, Lunghi M, et al. Flow cytometry evaluation of erythroid and myeloid dysplasia in patients with myelodysplastic syndrome. Leukemia 2005;19(5):776-83.

[18] Arber DA, Orazi A, Hasserjian R, et al. The 2016 revision to the World Health Organization classification of myeloid neoplasms and acute Leukemia. Blood 2016;127(20):2391-405. 